\title{
A NEW TAXONOMIC RANK FOR DROSERA PEDICELLARIS (DROSERACEAE)
}

JAN SCHLAUER • D-60594 Frankfurt/Main•Germany • jan@carnivorousplants.org

For consistency with a classification using infraspecific ranks (Schlauer, J. in Carniv. Pl. Newslett. 25: 72-75, 1996), the geographically overlapping and closely related taxa $D$. parvula (syn. $D$. minutiflora sensu auct. non Planch.: Lowrie, A. in Carnivorous Plants of Australia: Magnum Opus. Redfern Natural History Productions, Poole, p. 620, 2014) and D. pedicellaris are combined as varieties under the older species name. A new combination is thus proposed here.

Drosera parvula var. pedicellaris (Lowrie) Schlauer, comb. et stat. nov.

Basionym: Drosera pedicellaris Lowrie, Nuytsia 15: 59 (2002).

NAMES OF CARNIVOROUS PLANT CULTIVARS REGISTERED IN 2020

\begin{tabular}{|c|c|c|}
\hline Name & Publication & $\begin{array}{l}\text { Date of } \\
\text { registration }\end{array}$ \\
\hline Heliamphora 'Cyclops' A.Smith & Carniv. Pl. Newslett. 49: 141 (2020) & $23 \mathrm{Sep}$ \\
\hline H. 'Scylla' I.Bogdanow & Carniv. Pl. Newslett. 49: 93 (2020) & 19 Jun \\
\hline Sarracenia 'Arthur Wheeler' G.Wheeler & P1. Carniv. 40(2): 15 (2019) & 24 Jan \\
\hline S. 'Atlas 5' M.Srba \& M.King & Carniv. Pl. Newslett. 49: 91 (2020) & 19 Jun \\
\hline S. 'Christophe Maerten' L. Taerwe & Carniv. Pl. Newslett. 49: 92 (2020) & 19 Jun \\
\hline S. 'Glynis Wheeler' G.Wheeler & P1. Carniv. 40(2): 11 (2019) & 24 Jan \\
\hline S. 'Sarramphor' A.Fisch & Carniv. Pl. Newslett. 49: 49 (2020) & 21 May \\
\hline$S$. 'Super-duper' R.Ziemer & Carniv. Pl. Newslett. 49: 140 (2020) & $23 \mathrm{Sep}$ \\
\hline S. 'Wizzleberry Toad' J.Conner & Carniv. Pl. Newslett. 49: 50 (2020) & 21 May \\
\hline S. 'Yvaine Little' S.Little & Pl. Carniv. 40(2): 7 (2019) & 24 Jan \\
\hline Cephalotus 'OG Black' B.Lipinski & Carniv. Pl. Newslett. 49: 87 (2020) & 19 Jun \\
\hline Dionaea 'Axe' P.Mattevi & Carniv. Pl. Newslett. 48: 191 (2019) & 24 Jan \\
\hline D. 'CCCP Tasmanian Devil' C.Heath \& S.Wang & Carniv. Pl. Newslett. 49: 89 (2020) & 19 Jun \\
\hline D. 'FFT Stegosaurus' J.Moscoso & Carniv. Pl. Newslett. 48: 188 (2019) & 24 Jan \\
\hline$D$. 'Genepine' J.Mueller & Carniv. Pl. Newslett. 48: 189 (2019) & 24 Jan \\
\hline D. 'Morano' P.Mattevi & Carniv. Pl. Newslett. 48: 190 (2019) & 24 Jan \\
\hline D. 'Stove Fire' D.Weijie & Carniv. Pl. Newslett. 49: 142 (2020) & $23 \mathrm{Sep}$ \\
\hline Pinguicula 'Eye Spy' S.Bunclark & Carniv. Pl. Newslett. 49: 139 (2020) & $23 \mathrm{Sep}$ \\
\hline$P$. 'Razzberry Blonde' M.Rubnitz & Carniv. Pl. Newslett. 49: 90 (2020) & 19 Jun \\
\hline
\end{tabular}

